\title{
IMPACT OF WIND ENERGY INTEGRRATION ON ECONOMIC AND RELIABLE OPERATION OF ELECTRICAL NETWORS
}

\author{
A. A. Abou El-Ela*, S. M. Allam* and H. R. Somat** \\ *Electrical Engineering Dept, Faculty of Engineering, Minoufiya University, Shebin El-Kom, Egypt \\ ** Operation engineer, Egyptian Electricity Transmission Company, Egypt
}

\begin{abstract}
In this paper, an integration of a wind farm designed at the installation site of a new city with conventional power generation (CPG) is presented. Two scenarios are suggested to integrate this wind farm with CPG. The impact of this integration on the economic operation of the electrical network is studied using two proposed scenarios. Also, the impact of this integration on the reliable operation of the electrical network is introduced. A long-term load forecasting of the Egyptian electrical network is presented to be helpful in introducing the impact of wind farm integration on the reliable operation of the electrical network. The impact of wind energy integration on economic and reliable operation of electrical networks is applied to the new Borg El-Arab city in Egypt. The results show the capability of the two proposed scenarios.
\end{abstract}

Keywords : Economic Operation, Wind Farms, Conventional Power Generation, Interconnection of Power Networks, Reliability and Long-Term Load Forecasting.

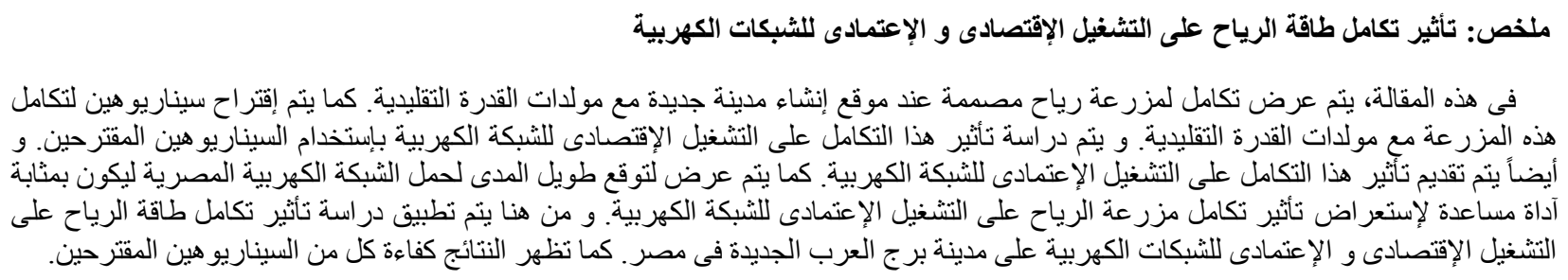

\section{Introduction}

The net effects of renewable energy sources (RESs) on the environment are far less problematic than those of nonrenewable energy sources [1]. Economic issues of wind energy systems are multidimensional. For a wind turbine, the fuel is free, but the capital investment is high. The variable production cost of wind is virtually zero and so with increasing wind penetration the total system fuel cost would be expected to fall. Hence, the savings in fuel costs for thermal plant arising from the installation of wind farms. On the other hand, the additional capital costs associated with integrating wind power generation into a conventional generation system may cause an increase in the overall cost that exceeds the savings in fuel costs [2].

The challenge facing a wind developer, and indeed any new generation resource, is whether it can interconnect to the electrical network or not [3]. Economically, the challenge facing the wind developer is to maintain the economic operation of the electrical network after the interconnection process because this process needs many interconnection requirements such as, it needs to two stages to raise wind generation voltage to the transmission voltage of the electrical network. Hence, the impact of electrical network interconnection of wind farms on economic operation of the electrical network should be studied. Also, another challenge facing the wind developer is to maintain the reliable operation of the electrical network after the interconnection process where this process have possible impacts on electric power system operations resulting from wind's variability and variations in electricity demand $[3,4]$.

In this paper, two scenarios are proposed to integrate a large scale wind farm nearby the new Borg El-Arab city in Egypt to feed its load with CPG. An economic investigation is introduced to show the best proposed scenario that the operator can choose according to the operating conditions of the power 


\section{A. A. Abou El-Ela, S. M. Allam and H. R. Somat " Impact of Wind Energy Integrati..."}

system. Also, the impact of electrical network interconnection of this wind farm on reliable operation of the electrical network is studied. A reliability study is carried out to find the capability of the wind energy to supply the electrical network of Egypt for two proposed operation scenarios.

\section{Problem Formulation}

Two processes are formulated to achieve the economic and reliable operation for power system as follows:

\subsection{Economic operation of wind farms with CPG}

Many studies were done on integration of RESs with CPG to study the impact of this integration on the required power from $\mathrm{CPG}$, fuel consumption and environment. Also, when large amounts of renewable energy are integrated with $\mathrm{CPG}$, it is very important to study the impact of this integration on the system economy [5 - 8]. Two proposed scenarios are presented as:

1) When the whole wind farm is totally designed and integrated with CPG.

2) When the wind farm is designed and integrated with CPG step by step according to the peak load forecasted of the new city for every year.

\subsubsection{Saving in total annual cost (TAC) of CPG}

Saving in TAC results from the integration of a large scale wind farm with CPG, because the load, which is required to be fed from CPG, will be fed from the wind farm instead of CPG [9]. TAC includes the annual capital cost (ACC) and the annual variable cost which includes the annual operation cost (AOC) and the annual fuel cost (AFC). AOC is very small with respect to $\mathrm{AFC}$, hence it can be neglected. Therefore, the annual variable cost can be represented as AFC only [10].

Annual capital cost of $\mathrm{CPG}\left(\mathrm{ACC}_{\mathrm{C}}\right)$ is obtained from the following equation:

$A C C_{C}=L_{f} * C_{c} * P_{L}$

Where, $\mathrm{L}_{\mathrm{f}}$ is the levelizing factor for conventional power stations and conventional fuel at the end-ofyear cost. $C_{c}$ is the ACC per $1 \mathrm{MW}$ of CPG, \$/MW. $\mathrm{P}_{\mathrm{L}}$ is the load which will be fed, MW.
The amount of the required fuel $\left(\mathrm{F}_{\mathrm{C}}\right)$ for $\mathrm{CPG}$ is given from the following equation:

$F_{C}=\left(K_{f} / H_{f}\right) *\left(E_{c} / \eta_{o}\right)$

Where, $\mathrm{K}_{\mathrm{f}}$ is the heat rate; $\mathrm{kcal} / \mathrm{MWh} . \mathrm{H}_{\mathrm{f}}$ is the heat value for 1 ton of $\mathrm{F}_{\mathrm{C}}, \mathrm{kcal} / \mathrm{ton}$. $\mathrm{E}_{\mathrm{c}}$ is the output energy of CPG; MWh. $\eta_{\mathrm{o}}$ is the overall efficiency of CPG.

The annual fuel cost of CPG $\left(\mathrm{AFC}_{\mathrm{C}}\right)$ is obtained from the following equation:

$A F C_{C}=L_{f} * C_{f} * F_{C}$

Where, $\mathrm{C}_{\mathrm{f}}$ is the cost per 1 ton of $\mathrm{F}_{\mathrm{C}}, \$ /$ ton.

Then, TAC for CPG $\left(\mathrm{TAC}_{\mathrm{C}}\right)$ is the sum of $\mathrm{ACC}_{\mathrm{C}}$ and $\mathrm{AFC}_{\mathrm{C}}$ which is obtained from the following equation:

$T A C_{C}=A C C_{C}+A F C_{C}$

\subsubsection{Integration of large scale wind farm with CPG}

The impact of this integration is determined in case of the previous two proposed scenarios.

\subsubsection{First proposed scenario}

In the first proposed scenario, a large scale wind farm is totally designed and integrated with CPG. The load will be fed and the surplus of the wind farm power will be delivered to the electrical network.

TAC of the wind farm $\left(\mathrm{TAC}_{\mathrm{WF}}\right)$ is given from the following equation:

$T A C_{W F}=N_{T} * T A C_{W G}$

Where, $\mathrm{TAC}_{\mathrm{WG}}$ is TAC of the wind generator. $\mathrm{N}_{\mathrm{T}}$ is the number of the whole wind farm turbines.

In order to make an electrical network interconnection for the wind farm, it is required to transform the generation voltage of wind generator to suitable medium voltage and then to the high voltage of the electrical network. Hence, step-up transformers are needed for this task [11]. The cost of these transformers certainly will affect on the economic operation of the overall system, where it will be added to the TAC of the wind farm. The number of step-up transformers $\left(\mathrm{N}_{\mathrm{Tr} 1}\right)$, which is required to transform the generator voltage to a suitable medium voltage, is equal to the number of wind generators 


\section{A. A. Abou El-Ela, S. M. Allam and H. R. Somat " Impact of Wind Energy Integrati..."}

and is divided into two parts. The first part is $\left(\mathrm{N}_{\mathrm{Tr} 1 \mathrm{a}}\right)$ which will feed the city load and the second part is $\left(\mathrm{N}_{\text {Trlb }}\right)$ which will feed the electrical network. Also, the number of step-up transformers $\left(\mathrm{N}_{\mathrm{Tr} 2}\right)$, which is required to transform the medium voltage to the high voltage of the electrical network, is determined according to the wind farm power which will be delivered to the electrical network and the power of the transformer which will be chosen. The actual number of these transformers $\left(\mathrm{N}_{\operatorname{Tr} 2 \text { (Act) }}\right)$ is the result of subtraction for the number of step-up transformers $\left(\mathrm{N}_{\mathrm{Tr} 2}\right)$ minus the number of transformers which feeds the city load now $\left(\mathrm{N}_{\operatorname{Tr} \text { (In Service) }}\right)$ which is calculated from the following equation:

$N_{T r 2(\text { Act })}=N_{T r 2}-N_{T r}($ In Service $)$

The costs of these transformers are the summation of the running cost and the fixed cost of them. The running costs of the transformers are the operation and maintenance costs that can be neglected because they are very small costs. The fixed costs of the transformers are the capital costs of it. Hence, the TAC of step-up transformer is the annual capital costs of it $\left(\mathrm{ACC}_{\mathrm{Tr}}\right)$ which are obtained from the following equation:

$A C C_{T r}=D R * C C_{T r}$

Where, $\mathrm{CC}_{\mathrm{Tr}}$ is the price of the transformer. DR is the annual discount rate and given by the following relation [12]:

$D R=r(1+r)^{n} /\left[(1+r)^{n}-1\right]$

Where, $\mathrm{r}$ is the interest rate. $\mathrm{n}$ is the life-time of the transformer.

Then, the total annual capital cost of the transformers $\left(\mathrm{ACC}_{\operatorname{Tr}(\mathrm{T})}\right)$ is obtained from the following equation:

$$
\begin{aligned}
A C C_{T r(T)}= & \left(N_{\operatorname{Tr} 1 a}+N_{\operatorname{Tr} 1 b}\right) * A C C_{\operatorname{Tr} 1} \\
& +N_{\operatorname{Tr} 2(A c t)} * A C C_{\operatorname{Tr} 2}
\end{aligned}
$$

From equations (4), (5) and (9), the net saving in CPG costs $\left(\mathrm{S}_{\mathrm{N}}\right)$ after adding the transformers is obtained from the following equation:

$$
S_{N}=T A C_{C}-\left(T A C_{W F}+A C C_{T r(T)}\right)
$$

The wind power saved $\left(\mathrm{P}_{\mathrm{ws}}\right)$, which will be delivered to the electrical network, is given from the following equation:
$P_{W S}=P_{W F}-P_{L}$

Where, $\mathrm{P}_{\mathrm{WF}}$ is the whole wind farm power.

The percentage of this power with respect to the whole wind farm power $\left(\mathrm{P}_{\mathrm{P}_{\mathrm{WS}}}\right)$ is obtained from the following equation:

$P_{P_{W S}}=\left(P_{W S} / P_{W F}\right) * 100$

\subsubsection{Second proposed scenario}

In the second proposed scenario, the wind farm is step by step designed and integrated with CPG according to the annual increasing of the load. This load will be fed and the surplus of the wind farm power will be delivered to the electrical network. The number of wind farm turbines for every year $\left(\mathrm{N}_{\mathrm{TA}}\right)$ is increased according to the load which is calculated from the following equation:

$N_{T A}=N_{T} * P_{L} / P_{W F}$

The total annual cost of the wind farm $\left(\mathrm{TAC}_{\mathrm{wf}}\right)$ is obtained from the following equation:

$T A C_{w f}=N_{T A} * T A C_{W G}$

In this scenario, the surplus of the wind farm power, which will be delivered to the electrical network, is very small and it can be neglected. Hence, the number of step-up transformers $\left(\mathrm{N}_{\mathrm{Tr}}\right)$ is the number of the transformers which is required to transform the generator voltage to the suitable medium voltage only and is equal to the number of wind generators that is required to feed the city load for every year and is increased according to the increasing of the load for every year. From equation (7), $\mathrm{ACC}_{\mathrm{Tr}}$ can be calculated taking into account the new price of the transformer for every year $\left(\mathrm{CC}_{\operatorname{Tr}}\right.$ (new) which is calculated from the following equation:

$C C_{T r \text { (new) }}=C C_{T r(\text { old })}+P_{\text {inc }} * C C_{T r \text { (old })}$

Where, $\mathrm{CC}_{\operatorname{Tr} \text { (old) }}$ is the price of the transformer in the previous year. $\mathrm{P}_{\mathrm{inc}}$ is the percentage increasing of the transformer price for every year.

$\mathrm{ACC}_{\mathrm{Tr}}$ (T) (new) for every year up to year 2035 is calculated from the following equation:

$$
\begin{aligned}
A C C_{\operatorname{Tr}(T)(\text { new })}= & A C C_{\operatorname{Tr}(T)(\text { old })} \\
& +N_{\operatorname{Tr}(\text { add })} * A C C_{\operatorname{Tr}(\text { new })}
\end{aligned}
$$




\section{A. A. Abou El-Ela, S. M. Allam and H. R. Somat " Impact of Wind Energy Integrati..."}

Where, $\mathrm{ACC}_{\operatorname{Tr}(\mathrm{T}) \text { (old) }}$ is the total annual capital cost of the transformers in the previous year. $\mathrm{N}_{\mathrm{Tr} \text { (add) }}$ is the number of the added transformers for every year. $\mathrm{ACC}_{\mathrm{Tr} \text { (new) }}$ is the annual capital cost of the added transformer.

From equations (4), (14) and (16), the net saving in CPG costs $\left(\mathrm{S}_{\mathrm{N}}\right)$ after adding the transformers is obtained from the following equation:

$S_{N}=T A C_{C}-\left(T A C_{w f}+A C C_{T r(T)(n e w)}\right)$

The wind farm power $\left(\mathrm{P}_{\mathrm{wf}}\right)$, according to the load for every year, is given from the following equation:

$P_{w f}=N_{T A} * P_{W G}$

Where, $\mathrm{P}_{\mathrm{WG}}$ is the average output power of the wind generator.

The wind power saved, which will be delivered to the electrical network, is given from the following equation:

$P_{W S}=P_{w f}-P_{L}$

Also, the percentage of this power with respect to the whole wind farm power is obtained from the following equation:

$P_{P_{W S}}=\left(P_{W S} / P_{w f}\right) * 100$

\subsection{Impact of wind farms integration on reliable operation of the electrical network}

The impact of the wind farms integration on reliable operation of the electrical network can be determined by calculating the percentage improvement of reliability for the two previous proposed scenarios. The percentage improvement of reliability of the electrical network is the ratio between the wind power, which will be delivered to the electrical network, and the electrical network power [13].

\subsubsection{Electrical network peak load forecasting}

The electrical network peak load can be estimated for a group of years in the future by using load forecasting techniques. The basic concept of these techniques depends on the existence of the historical data of the peak load of the electrical network for a group of years in the past. Then, these data can be represented by a suitable mathematical equation according to the nature of the electrical loads increasing on the network. Knowing this equation, the peak load for the electrical network for a group of years in the future can be estimated. Extrapolation of trend curves technique as a conventional technique and genetic algorithm (GA) and particle swarm optimization (PSO) technique as modern techniques of long-term load forecasting with linear and exponential regression models can be used for this purpose.

\subsubsection{Percentage improvement of the electrical network reliability}

The percentage improvement of the electrical network reliability can be calculated for the two previous proposed scenarios as:

i. First proposed scenario

In this proposed scenario, the generated power from the wind farm is very large with respect to the city load, especially in the first years of integration. Hence, the percentage improvement of the electrical network reliability will be large. This percentage is calculated from the following equation:

$\operatorname{PIENR}(\%)=\left[\left(P_{W F}-P_{F L}\right) / P_{F L N}\right] * 100$

Where, $\mathrm{P}_{\mathrm{WF}}$ is the total wind farm power, $\mathrm{P}_{\mathrm{FL}}$ is the forecasted load of the city and $\mathrm{P}_{\mathrm{FLN}}$ is the forecasted peak load of the electrical network for every year.

\section{ii. Second proposed scenario}

In this proposed scenario, the amount of wind power, which will be delivered to the electrical network, will be very small and the percentage improvement of the electrical network reliability also will be closed to zero. Then, it can be deduced that there is no improvement in the electrical network reliability in this proposed scenario.

\section{Applications}

In this paper, the impact of integration of a large scale wind farm at the new Borg El-Arab city with CPG on the economic and reliable operation of the overall system using two proposed scenarios is studied.

\subsection{Economic operation of wind farms with CPG}

The net saving in CPG costs and the amount of the wind power saved, which will be delivered to the Egyptian electrical network, after feeding the new 


\section{A. A. Abou El-Ela, S. M. Allam and H. R. Somat " Impact of Wind Energy Integrati..."}

city for the two previous proposed scenarios are calculated considering the following main data for the chosen transformers [14]:

- $\operatorname{Tr}_{1}: 1.05 \mathrm{MVA}, 0.69 / 22 \mathrm{KV}$ and its price is equal to $26666.667 \$$.

- $\operatorname{Tr}_{2}: 25 \mathrm{MVA}, 22 / 66 \mathrm{KV}$ and its price is equal to 833333.333 \$.

\subsubsection{Annual cost saving calculation in TAC for CPG}

Equations from (1) to (4) are applied considering the following values:

- The value of $L_{f}$ in equations (1) and (3) is taken as 1.05 and 1.1 , respectively.

- The values of $\mathrm{C}_{\mathrm{c}}$ and $\mathrm{C}_{\mathrm{f}}$ in equations (1) and (3) are taken as $1800 * 10^{3} \$ / \mathrm{MW}$ [15] and 100 $\$ /$ ton, respectively.

- The values of $K_{f}, H_{f}$ and $\eta_{o}$ in equation (2) are taken as $860 * 10^{3} \mathrm{kcal} / \mathrm{MWh}, 11500 * 10^{3}$ $\mathrm{kcal} / \mathrm{ton}$ and 0.55 , respectively.

- The values of $\mathrm{P}_{\mathrm{L}}$ in equation (1) are the forecasted peak load demand for the new Borg El-Arab city $\left(\mathrm{P}_{\mathrm{FL}}\right)$ from year 2012 to year 2035.

- The values of $E_{c}$ in equation (2) are the output energy of $\mathrm{CPG}$ corresponding to $\mathrm{P}_{\mathrm{FL}}$ for every year.

The annual cost saving in TAC for CPG from year 2012 to year 2035 is given in Fig. (1). From this Figure, it can be found that the annual cost saving in TAC for CPG is increased with increasing of the forecasted peak load demand of this city. This is because of the increasing of the forecasted peak load demand of this city for every year, which will be fed from the wind farm instead of CPG.

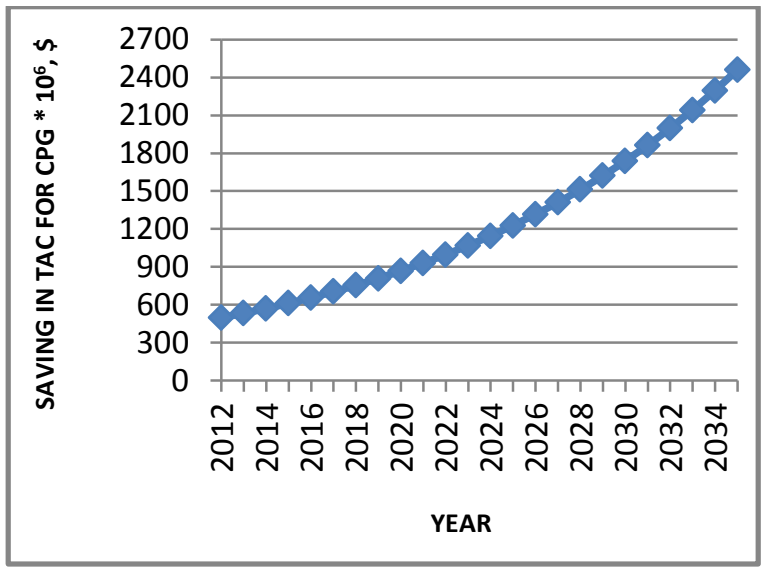

Fig. (1) Annual cost saving in TAC for CPG from year 2012 to year 2035 without wind farm integration

\subsubsection{Calculations of first proposed scenario}

The whole wind farm, which will be integrated with CPG, gives 1262.195 MW with number of wind turbine generators of 1853 units. Also, the TAC of the wind generator used is equal to $354480 \$$. From the equations from (5) to (10), the TAC for the whole wind farm is $656851440 \$$ and the annual net costs saving in the TAC for CPG after adding the transformers cost from year 2012 to year 2035 is shown in Fig. (2). From this Figure, it can be found that there is no net cost saving in the first five years because the construction cost of the wind farm and the transformers cost are more expensive than the cost saving of CPG for these years.

Also, the percentage of the wind power, which will be delivered to the Egyptian electrical network with respect to the whole wind farm power, is calculated by using the equations (11) and (12) and is shown in Fig. (3). From this Figure, it can be found that the highest percentage of the wind power, which will be delivered to the Egyptian electrical network, is in the first year of 2012 and this percentage is decreased to reach the lowest percentage in the year of 2035. This is because the forecasted peak load demand of the new city, which is totally fed from the wind farm, is increased through the load forecasted period $(2012-2035)$ and the wind power, which will be delivered to the Egyptian electrical network, is decreased. 


\section{A. A. Abou El-Ela, S. M. Allam and H. R. Somat " Impact of Wind Energy Integrati..."}

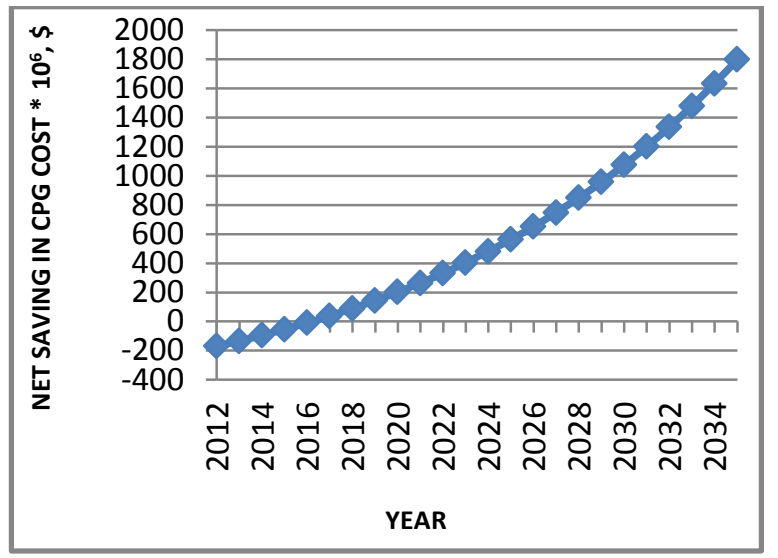

Fig. (2) Annual net costs saving in TAC for CPG from year 2012 to year 2035 using the first proposed scenario

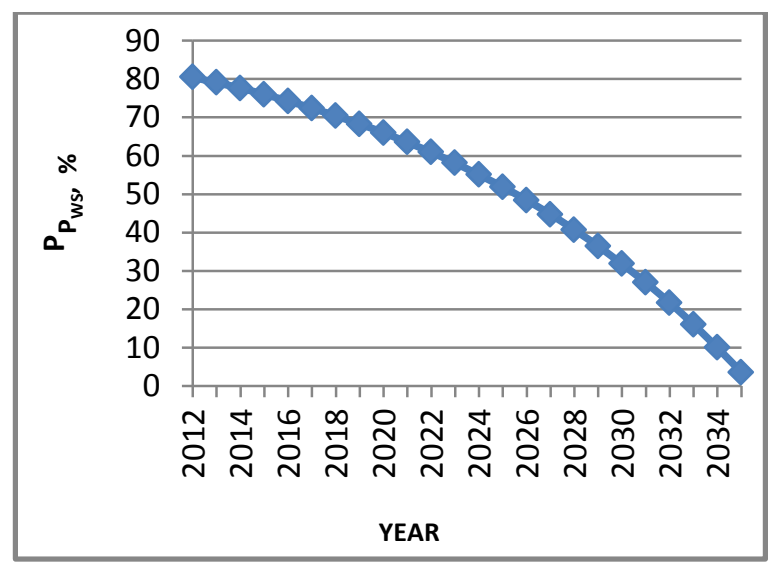

Fig. (3) Percentages of wind power which will be delivered to the Egyptian electrical network from year 2012 to year 2035 using the first proposed scenario

\subsubsection{Calculations of second proposed scenario}

In this proposed scenario, the number of the wind turbine generators for every year through the load forecasted period $(2012$ - 2035) must be determined at the beginning of calculations. The number of step-up transformers is equal to the number of wind generators which is required to feed the city load for every year. From the equations from (13) to (17), the annual net costs saving in the TAC for CPG after adding the transformers cost from year 2012 to year 2035 is shown in Fig. (4). From this Figure, it can be found that there is a large net costs saving is obtained from the first year of the load forecasted period 2012. This net costs saving is increased according to the increasing of the peak load forecasted of the new city.

The percentages of the wind power, which will be delivered to the Egyptian electrical network with respect to the whole wind farm power, are determined by using the equations (18), (19) and (20). The average output power of the wind generator used in equation (18) is equal to $681.163 \mathrm{~kW}$. The percentages of wind power, which will be delivered to the Egyptian electrical network up to year 2035, are shown in Fig. (5). From this Figure, it can be found that the general behavior of these percentages is decreased for the load forecasted period. This is because the number of wind turbine generators is closely chosen according to the peak load forecasted of the new city.

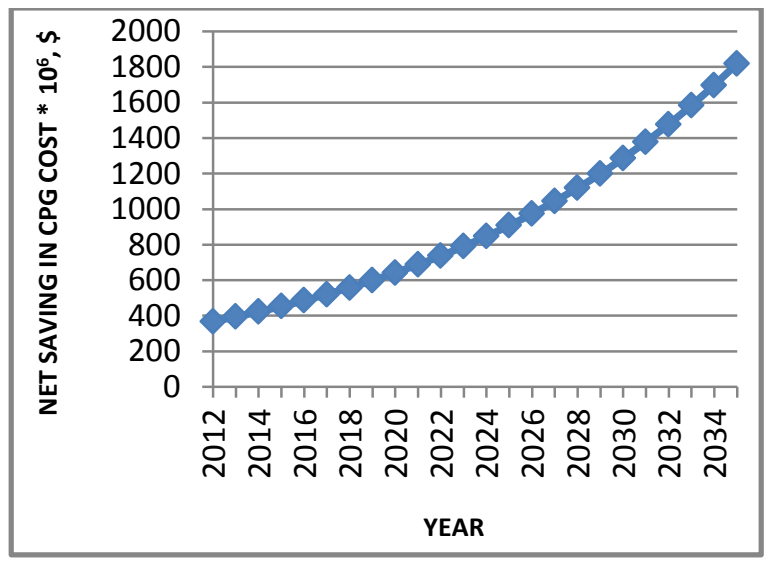

Fig. (4) Annual net costs saving in TAC for CPG from year 2012 to year 2035 using the second proposed scenario

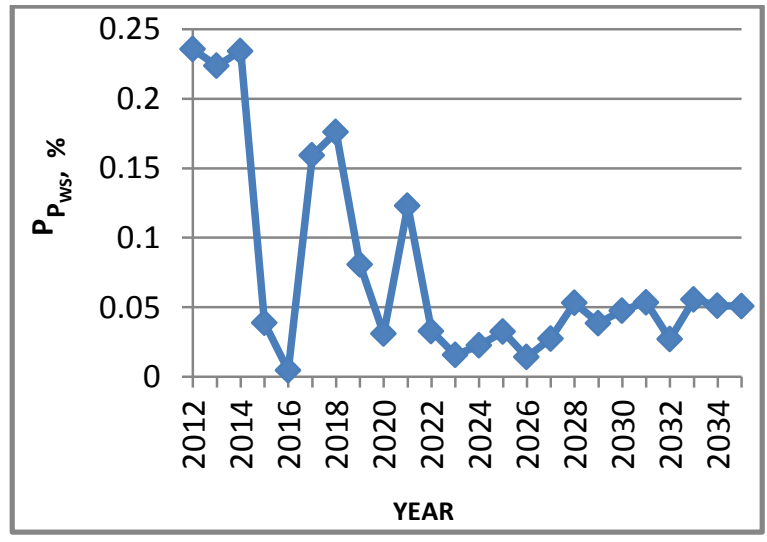

Fig. (5) Percentages of wind power which will be delivered to the Egyptian electrical network from year 2012 to year 2035 using the second proposed scenario 


\section{A. A. Abou El-Ela, S. M. Allam and H. R. Somat " Impact of Wind Energy Integrati..."}

\subsubsection{A comparison between results of two proposed scenarios}

Fig. (6) shows a comparison between the net costs saving in TAC for CPG for the two proposed scenarios from year 2012 to year 2035. From this Figure, the total net costs saving in TAC for CPG up to year 2035 using the second proposed scenario is greater than that for the first proposed scenario. Also, a comparison between the percentages of the wind power, which will be delivered to the Egyptian electrical network with respect to the whole wind farm power for the two proposed scenarios, are obtained as shown in Fig. (7). From this Figure, these percentages using the first proposed scenario are larger than that using the second proposed scenario, which are extremely small.

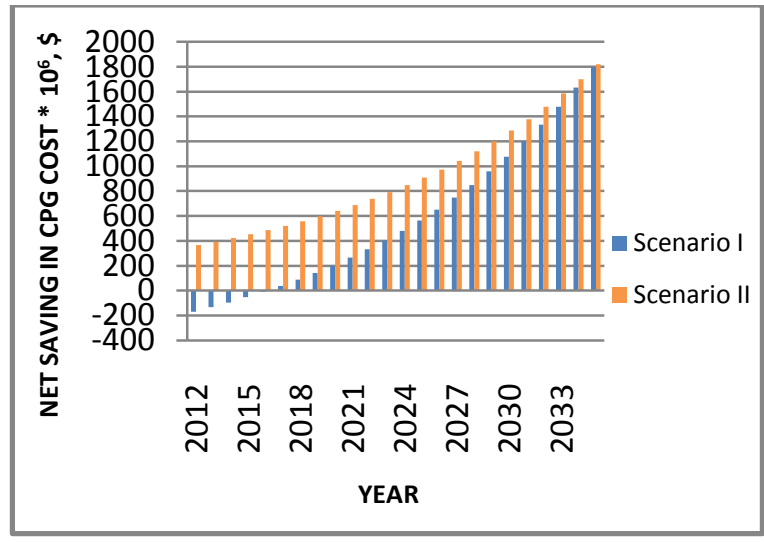

Fig. (6) A comparison between the net costs saving in TAC for CPG for two proposed scenarios from year 2012 to year 2035

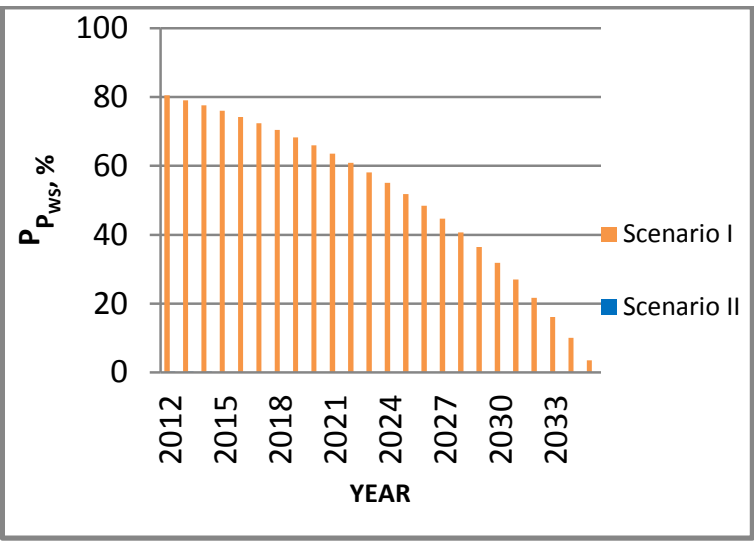

Fig. (7) A comparison between the percentages of the wind power, which will be delivered to the Egyptian electrical network from year 2012 to year 2035

\subsection{Impact of wind farms integration on reliable operation of the electrical network}

The impact of electrical network integration of the wind farm designed nearby new Borg El-Arab city on the reliable operation of the Egyptian electrical network is determined by calculating the percentage improvement of reliability of the Egyptian electrical network. This percentage is the ratio between the wind power, which will be delivered to the Egyptian electrical network, and the Egyptian electrical network power. The Egyptian electrical network power is the peak load of the Egyptian electrical network which should be forecasted at first as shown in details below.

\subsubsection{Egyptian electrical network peak load forecasting}

The historical data of the Egyptian electrical network peak load from year 2003 to year 2011 is shown from Table (1) [10]. The extrapolation of trend curves technique as a conventional technique and GA and PSO technique as modern techniques of longterm load forecasting techniques with linear and exponential regression models are applied using the historical data as shown in Table (1), the peak load of the Egyptian electrical network from year 2012 to year 2035 is obtained in Table (2). From this Table, it can be found that the peak load of the Egyptian electrical network which is obtained from GA with the exponential increasing form are the best results and will be chosen to apply because it is more accurate compared to the actual peak load of the Egyptian electrical network from year 2012 to year 2015 as shown in Table (3).

Table (1) Historical data of the Egyptian electrical network peak load

\begin{tabular}{|c|c|}
\hline Year & $\mathrm{P}_{\mathrm{LN}}(\mathrm{GW})$ \\
\hline 2005 & 16.019 \\
\hline 2006 & 17.179 \\
\hline 2007 & 18.385 \\
\hline 2008 & 19.400 \\
\hline 2009 & 20.270 \\
\hline 2010 & 21.137 \\
\hline 2011 & 21.801 \\
\hline
\end{tabular}




\section{A. A. Abou El-Ela, S. M. Allam and H. R. Somat " Impact of Wind Energy Integrati..."}

Table (2) Peak load forecasting of the Egyptian electrical network from year 2012 to year 2035

\begin{tabular}{|c|c|c|c|c|c|c|}
\hline \multirow[b]{2}{*}{ Year } & \multicolumn{2}{|c|}{ Extrapolation } & \multicolumn{2}{|c|}{ Genetic Algorithm } & \multicolumn{2}{|c|}{$\begin{array}{c}\text { Particle Swarm } \\
\text { Optimization }\end{array}$} \\
\hline & $\begin{array}{c}P_{\text {FLN }} \\
\text { Linear } \\
(\mathrm{GW})\end{array}$ & \begin{tabular}{|c|}
$P_{\mathrm{FLN}}$, \\
Exponential \\
$(\mathrm{GW})$
\end{tabular} & \begin{tabular}{|c|}
$P_{\text {FLN }}$ \\
Linear \\
$(G W)$ \\
\end{tabular} & \begin{tabular}{|c|}
$P_{\mathrm{FLN}}$, \\
Exponential \\
$(\mathrm{GW})$
\end{tabular} & \begin{tabular}{|c|}
$P_{\text {FLN }}$ \\
Linear \\
$(\mathrm{GW})$ \\
\end{tabular} & \begin{tabular}{|c}
$P_{\mathrm{FLN}}$, \\
Exponential \\
$(\mathrm{GW})$
\end{tabular} \\
\hline 2012 & 23.048 & 23.414 & 23.342 & 24.070 & 23.864 & 24.537 \\
\hline 2013 & 24.018 & 24.646 & 24.387 & 25.512 & 25.038 & 26.133 \\
\hline 2014 & 24.987 & 25.944 & 25.433 & 27.040 & 26.211 & 27.833 \\
\hline 2015 & 25.957 & 27.310 & 26.478 & 28.660 & 27.385 & 29.644 \\
\hline 2016 & 26.926 & 28.748 & 27.524 & 30.378 & 28.558 & 31.572 \\
\hline 2017 & 27.896 & 30.262 & 28.569 & 32.198 & 29.732 & 33.626 \\
\hline 2018 & 28.866 & 31.855 & 29.614 & 34.127 & 30.905 & 35.814 \\
\hline 2019 & 29.835 & 33.533 & 30.660 & 36.172 & 32.079 & 38.144 \\
\hline 2020 & 30.805 & 35.298 & 31.705 & 38.339 & 33.252 & 40.625 \\
\hline 2021 & 31.774 & 37.157 & 32.751 & 40.636 & 34.426 & 43.268 \\
\hline 2022 & 32.744 & 39.114 & 33.796 & 43.071 & 35.599 & 46.083 \\
\hline 2023 & 33.713 & 41.173 & 34.842 & 45.651 & 36.772 & 49.081 \\
\hline 2024 & 34.683 & 43.341 & 35.887 & 48.386 & 37.946 & 52.274 \\
\hline 2025 & 35.652 & 45.623 & 36.933 & 51.285 & 39.119 & 55.675 \\
\hline 2026 & 36.622 & 48.025 & 37.978 & 54.358 & 40.293 & 59.296 \\
\hline 2027 & 37.591 & 50.554 & 39.024 & 57.615 & 41.466 & 63.154 \\
\hline 2028 & 38.561 & 53.216 & 40.069 & 61.067 & 42.640 & 67.263 \\
\hline 2029 & 39.530 & 56.018 & 41.114 & 64.726 & 43.813 & 71.639 \\
\hline 2030 & 40.500 & 58.968 & 42.160 & 68.604 & 44.987 & 76.299 \\
\hline 2031 & 41.470 & 62.073 & 43.205 & 72.714 & 46.160 & 81.263 \\
\hline 2032 & 42.439 & 65.341 & 44.251 & 77.071 & 47.334 & 86.549 \\
\hline 2033 & 43.409 & 68.781 & 45.296 & 81.689 & 48.507 & 92.180 \\
\hline 2034 & 44.378 & 72.403 & 46.342 & 86.583 & 49.681 & 98.177 \\
\hline 2035 & 45.348 & 76.215 & 47.387 & 91.771 & 50.854 & 104.564 \\
\hline
\end{tabular}

Table (3) Actual and forecasted peak load of the Egyptian electrical network from year 2012 to year 2015

\begin{tabular}{|c|c|c|c|c|}
\hline Year & 2012 & 2013 & 2014 & 2015 \\
\hline $\begin{array}{c}\text { Actual peak load } \\
\text { demand, GW }\end{array}$ & 23.88 & 24.86 & 26.69 & 28.19 \\
\hline $\begin{array}{c}\text { Forecasted peak load } \\
\text { demand, GW }\end{array}$ & 24.070 & 25.512 & 27.040 & 28.660 \\
\hline
\end{tabular}

\subsubsection{Percentage improvement of the Egyptian electrical network reliability using the first proposed scenario}

The percentage improvement of the Egyptian electrical network reliability is calculated using the equation (21). Fig. (8) shows the variation of this percentage from year 2012 to year 2035. From this Figure, it can be found that the highest value of the percentage improvement of the Egyptian electrical network reliability is in the first year of 2012, while the lowest value of this percentage is in the last year of 2035. It means that the percentage improvement of the Egyptian electrical network reliability depends on the amount of wind power which will be delivered to the Egyptian electrical network for every year. This power depends on the value of the increasing of the forecasted peak load demand of the city for every year. From this result, it can be shown that the wind farm designed nearby new Borg El-Arab city will increase the percentage improvement of the Egyptian electrical network reliability because the ability of this network to face any increasing of loads for every year will increase.

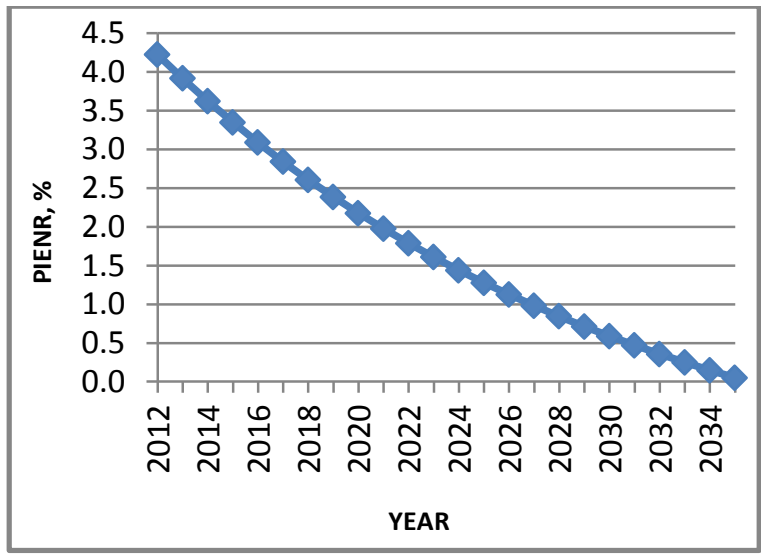

Fig. (8) Percentages improvement of the Egyptian electrical network reliability from year 2012 to year 2035

\section{Conclusions}

In this paper, the possibility of operating wind energy with the main conventional supply (substation) to supply new cities has been discussed. Also, the impact of integration of wind energy on the economic and reliable operation of the Egyptian electrical network have been discussed and analyzed. From this study, the following conclusions have been deduced:

1. The impact of a large scale wind farm with CPG nearby new Borg El-Arab city on the economical side of the overall system for the two proposed scenarios has been determined by calculating the net costs saving in TAC for CPG. It has been deduced that the total net costs saving in TAC for CPG for the forecasted period using the first 


\section{A. A. Abou El-Ela, S. M. Allam and H. R. Somat " Impact of Wind Energy Integrati..."}

proposed scenario is less than that using the second proposed scenario.

2. The percentage of the wind power, which will be delivered to the Egyptian electrical network, has been obtained for the two proposed scenarios. It has been deduced that the percentage of the wind power, which will be delivered to the Egyptian electrical network using the first proposed scenario, has been extremely larger than that using the second proposed scenario.

3. The impact of wind energy on the reliable operation of the Egyptian electrical network has been determined for the two proposed scenarios. This impact has been determined by calculating the percentage improvement of the Egyptian electrical network reliability. It can be deduced that the Egyptian electrical network will be more reliable using the first proposed scenario than that using the second proposed scenario.

Therefore, the electrical network operators can trade-off between the two proposed scenarios according to the operating conditions of the power system. It means that if the operator wants to obtain a large net costs saving in TAC for CPG and the percentage of the wind power, which will be delivered to the Egyptian electrical network, is not important for him, he should choose the second proposed scenario. On the other hand, if he wants to obtain a large percentage of the wind power, which will be delivered to the Egyptian electrical network, and the net costs saving in TAC for CPG is not important for him, he should choose the first proposed scenario.

\section{References}

[1] "Wind Power", Article, Wikipedia, the free encyclopedia, Available online at https://en.wikipedia.org/wiki/Wind_power.

[2] "Impact of Wind Power Generation in Ireland on the Operation of Conventional Plant and the Economic Implications", Report, ESB National Grid, Available online at www.eirgrid.com, 2004.

[3] "Transmission and Wind Energy: Capturing the Prevailing Winds for the Benefit of Customers", National Grid, Available online at www.nationalgridus.com, 2006.

[4] "Grid Integration", Article, Department of Energy, Office of Energy Efficiency \&
Renewable Energy, Available online at http://energy.gov/eere/wind/grid-integration.

[5] B. C. Ummels, "Power System Operation with Large-Scale Wind Power in Liberalised Environments", Book, Labor Grafimedia bv, Utrecht, 2008.

[6] D. Justus, "Case Study 5: Wind Power Integration into Electricity Systems", Research, Organisation for Economic Co-operation and Development, International Energy Agency, 2005.

[7] J. Charles Smith, M. R. Milligan, E. A. DeMeo and Brian Parsons, "Utility Wind Integration and Operating Impact State of the Art", IEEE Transactions on Power Systems, Vol. 22, No. 3, pp. 900 - 908, 2007.

[8] J. Charles Smith, Edgar A. DeMeo, Brian Parsons and Michael Milligan, "Wind Power Impacts on Electric Power System Operating Costs: Summary and Perspective on Work to Date", Global Wind Power Conference Chicago, Illinois, 2004.

[9] A. Ntomaris and A. Bakirtzis, "Stochastic Scheduling of Hybrid Power Stations in Insular Power Systems With High Wind Penetration", IEEE, Transactions on Power Systems, 2015.

[10] A. Abou El-Ela, A. El-Zeftawy, S. Allam, and G. Atta, "Long-Term Load Forecasting and Economical Operation of Wind Farms for Egyptian Electrical Network”, Vol. 79, pp. 1032 - 1037, International Journal of Electric Power Systems Research (EPSR), 2009.

[11] J. Daniel, Sh. Liu, E. Ibanez, K. Pennock, G. Reed and S. Hanes, "National Offshore Wind Energy Grid Interconnection Study, Executive Summary", Report, ABB, Inc., 2014.

[12] E. Feinberg and D. Genethliou, "Applied Mathematics for Restructured Electric Power Systems", Book, pp. 269 - 285, Springer Science \& Business Media, Inc., 2005.

[13] I. Adebayo, A. Olaomi, and E. Buraimoh, "Power System Reliability Analysis Incorporating Distributed Generator", International Journal of Scientific \& Engineering Research, Vol. 4, No. 3, 2013.

[14] "The General Administration of Electricity", Data, the Administrative Apparatus of the new 
A. A. Abou El-Ela, S. M. Allam and H. R. Somat " Impact of Wind Energy Integrati..."

Borg El-Arab City, Urban Communities Authority, 2011.

[15] S. Kaplan, "Power Plants: Characteristics and Costs", Report, Congressional Research Service, 2008. 